\title{
Le choc toxinique streptococcique
}

\section{Streptococcal toxic shock syndrome}

\section{A. Beltramini $\cdot$ K. Bouferrache $\cdot$ M. Pessoa $\cdot$ D. Pateron}

Reçu le 20 septembre 2011 ; accepté le 29 novembre 2011

(C) SFMU et Springer-Verlag France 2011

Résumé Les infections streptococciques graves et le choc toxinique streptococcique (CTS) ont une incidence croissante. Le diagnostic demeure difficile devant la relative rareté du CTS et la banalité des symptômes initiaux. L'évolution est rapidement sévère et le taux de mortalité est élevé. Le streptocoque A $\beta$-hémolytique (SAH) possède de nombreux facteurs de virulence, constitutionnels et sécrétés, lui permettant une adhésion, une invasion tissulaire et lui conférant une résistance au système de défense de l'hôte. Il existe une population à risque identifiée à laquelle se surajoute la femme dans la période du péripartum. Il est indispensable d'y penser devant tout état de choc au cours du troisième trimestre d'une grossesse. Le traitement actuel repose sur la réanimation du choc, l'administration d'antibiotiques à doses élevées en monothérappie ou en association et sur la prise en charge chirurgicale du site infecté. L'administration d'immunoglobulines polyclonales a été proposée avec des résultats intéressants en termes de survie. La recherche vaccinale sur le SAH est un important espoir pour réduire la mortalité du choc streptococcique. Pour citer cette revue : Ann. Fr. Med. Urgence 2 (2012).

Mots clés Choc toxinique $\cdot$ Streptococcus pyogènes Exotoxines $\cdot$ Immunoglobulines

\author{
A. Beltramini \\ Samu 28, CHG Victor-Jousselin, F-28100 Dreux, France \\ K. Bouferrache \\ Réanimation médicale, CHU Ambroise-Paré, \\ AP-HP, F-92100 Boulogne-Billancourt, France \\ M. Pessoa \\ Samu 78, CHR André-Mignot, F-78150 Le-Chesnay, France \\ D. Pateron $(\bowtie)$ \\ Service d'accueil des urgences, CHU Saint-Antoine, \\ AP-HP, F-75012 Paris, France \\ e-mail : dominique.pateron@sat.aphp.fr
}

Université Pierre-et-Marie-Curie-Paris-VI, F-75005 Paris, France

\begin{abstract}
There is an increasing incidence of serious streptococcal infections and streptococcal toxic shock syndrome. Diagnosis remains difficult, due to the relative rarity of streptococcal toxic shock syndrome and the trivial nature of the initial symptoms. Patients deteriorate rapidly and the mortality rate is high. Group A $\beta$-haemolytic streptococci possess numerous virulence factors, both constitutional and secreted, allowing adhesion, invasion of tissue and which provide a resistance to the host's defence system. There is an identified at-risk population, which includes women in the peripartum period. It is essential to give prior consideration to any type of shock during the third trimester of pregnancy. The current treatment given to combat shock is the administration of high-dose antibiotics, either as a single therapy or in conjunction with surgical management of the infected site. Polyclonal immunoglobulin administration has been proposed, with interesting results in terms of survival rates. Research into a Group A $\beta$-haemolytic streptococci vaccine is of significance in the reduction of mortality from streptococcal shock. To cite this journal: Ann. Fr. Med. Urgence 2 (2012).
\end{abstract}

Keywords Toxic shock syndrome $\cdot$ Streptococcus pyogenes $\cdot$ Exotoxins $\cdot$ Immunoglobulins

\section{Introduction}

Une augmentation des cas d'infection streptococcique grave (ISG) a été recensée depuis 20 ans [1]. L'incidence des ISG varie entre 1,5 et 20 cas pour 100000 habitants par an en fonction des études [1-8]. Parmi ces ISG, 10 à $14 \%$ développeront un choc toxinique streptococcique (CTS). Au cours d'une ISG, la survenue d'un CTS grève le pronostic vital du patient. La mortalité s'élève alors à 30-81\% malgré les thérapeutiques actuelles [9-11]. En 1993, des critères diagnostiques ont été proposés par un groupe de travail sur les infections streptococciques. Toutefois, le CTS reste sousdiagnostiqué et reconnu tardivement en raison de sa rareté et 
de la banalité des symptômes initiaux. Le streptocoque A $\beta$-hémolytique (SAH) possède de multiples facteurs de virulence qui vont générer des réactions immunologiques complexes et vont être à l'origine du CTS. Des facteurs de risque ont été identifiés, mais les nombreuses publications de CTS pendant le péripartum permettent d'isoler une nouvelle population à risque : la femme au troisième trimestre d'une grossesse ou en post-partum. Afin d'améliorer la survie de patients présentant un CTS, le traitement doit être « agressif» et instauré le plus précoce possible. Devant la persistance d'un taux de mortalité précocement élevé, d'autres thérapeutiques ont été évaluées, notamment les immunoglobulines polyclonales (IgG). Enfin, le développement d'un vaccin nourrit d'importants espoirs pour réduire l'incidence du CTS et réduire la mortalité.

\section{Épidémiologie}

Jusqu'au début du $\mathrm{xx}^{\mathrm{e}}$ siècle, la scarlatine était une maladie infantile redoutable avec un taux de mortalité élevé. L'amélioration des conditions de vie, les progrès médicaux et l'avènement des antibiotiques ont transformé le pronostic des infections streptococciques et ont réduit les risques de rhumatisme articulaire aigu et de glomérulonéphrite poststreptococcique. Au cours des années 1980, des cas plus fréquents et plus graves d'infections streptococciques (ISG) ont été rapportés [1]. Les ISG restent relativement rares $(0,3$ à 5 cas pour 100000 habitants). Il semble qu'un gradient Nord-Sud existe [2-8]. Actuellement, 10 à $14 \%$ des ISG se compliquent d'un CTS (Tableau 1). Le CTS s'accom-

Tableau 1 Incidence des infections graves à streptocoque A $\beta$-hémolytique et du CTS

\begin{tabular}{|lll|}
\hline & $\begin{array}{l}\text { Nombre d'ISG } \\
\text { pour 100 000 } \\
\text { habitants par an }\end{array}$ & Taux de CTS (\%) \\
\hline États-unis [7] & $3,5-4,4$ & 12 \\
Canada [2] & $1,5-5,2$ & 14 \\
Danemark [5] & 2,6 & 10 \\
Suède [4] & 3 & 11 \\
Finlande [9] & 2,3 & ND \\
Angleterre [12] & 2,9 & ND \\
France [10] & $1,2-2,7$ & 19 \\
Tchécoslovaquie [6] & 1,5 & 13 \\
Italie [6] & 0,4 & ND \\
Roumanie [6] & 0,4 & ND \\
Chypre [6] & 0,3 & ND \\
\hline ND : non disponible ; ISG : infection streptococcique grave ; \\
CTS : choc toxinique streptococcique.
\end{tabular}

pagne d'un fort taux de mortalité entre 30 et $81 \%$ selon les études $[1,2,4,5,7]$. Chez des patients hospitalisés en réanimation pour une ISG, le développement d'un CTS est un facteur indépendant de surmortalité [13]. Le pronostic du CTS ne semble pas être lié au niveau de soins du pays.

Les raisons de cette recrudescence ne sont pas claires. L'émergence de souches streptococciques de virulence accrue, l'identification de facteurs de virulence et la découverte de mécanismes immunologiques complexes apportent des éléments de réponse.

\section{Physiopathologie du CTS}

\section{Classifications et SAH}

Le SAH ou streptocoque pyogène est une bactérie cocci Gram positive, immobile, asporulée qui génère une hémolyse complète (type $\beta$ ) sur une gélose de sang. Son métabolisme est anaérobie et son développement est favorisé par le dioxyde de carbone, mais elle tolère un milieu contenant de l'oxygène. Il appartient au sérogroupe A de la classification de Lancefield.

Les sérotypes sont déterminés grâce à la constitution antigénique des protéines de sa paroi : protéines $M, T, R, F \ldots$ L'identification du sérotype $\mathrm{M}$, parmi les 150 possibles, est primordial, car il est associé à des degrés de virulence variables.

\section{Facteurs de virulence}

La virulence du SAH n'est pas totalement élucidée. Le SAH possède un arsenal lui permettant une adhésion, une invasion, lui conférant une protection contre les défenses de l'hôte, et favorisant l'évolution vers l'état de choc du patient infecté (Fig. 1) [14].

\section{Facteurs de virulence constitutionnels}

Le SAH possède des facteurs de virulence constitutionnels répartis au sein des trois couches de sa paroi.

La capsule, constituée d'acide hyaluronique, empêcherait la phagocytose, favoriserait l'adhésion du SAH et l'invasion tissulaire par l'intermédiaire des récepteurs cellulaires à la hyaluronidase (CD44) [15].

Les protéines $M$, enchâssées dans la paroi, sont codées par le gène Emm et confèrent une virulence spécifique au streptocoque. Elles favoriseraient la fixation de la bactérie au niveau de la muqueuse pharyngée et de la peau. Elles inhiberaient la phagocytose et seraient responsables d'une résistance à l'opsonisation en inactivant la voie du complément. Elles empêcheraient l'action des polynucléaires neutrophiles [14] et interviendraient dans des mécanismes 


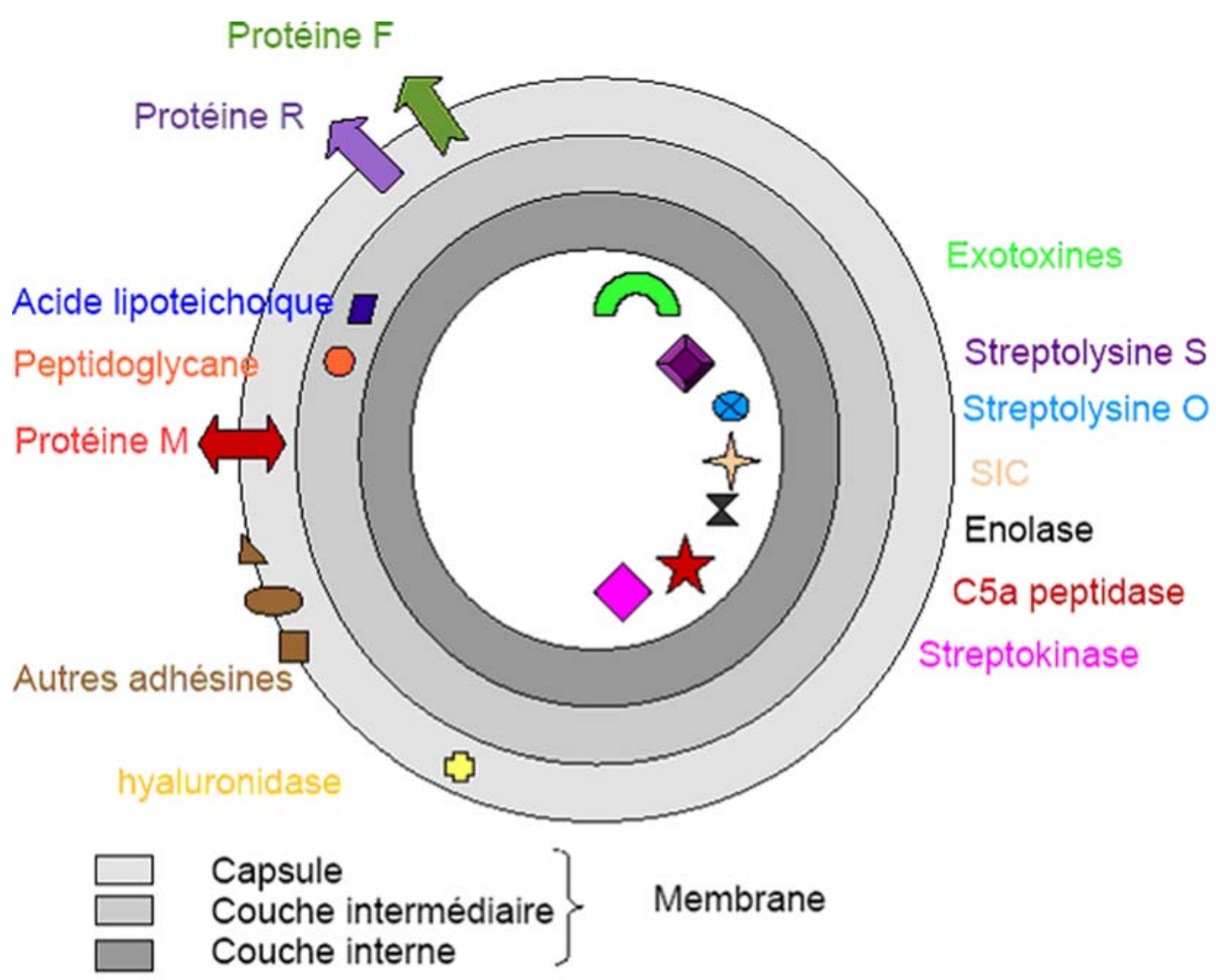

\begin{tabular}{|l|l|}
\hline $\begin{array}{l}\text { Résistance aux défenses } \\
\text { immunitaires }\end{array}$ & \\
\hline Phagocytose & $\begin{array}{l}\text { Capsule } \\
\text { Protéine M } \\
\text { Streptolysine O }\end{array}$ \\
\hline Complément & $\begin{array}{l}\text { C5a peptidase } \\
\text { SIC } \\
\text { Acide lipoteichoique }\end{array}$ \\
\hline Défaut immunisation & Proteine M \\
\hline $\begin{array}{l}\text { Inhibition polynucléaire } \\
\text { neutrophile }\end{array}$ & $\begin{array}{l}\text { Streptolysine S } \\
\text { Protéine M }\end{array}$ \\
\hline
\end{tabular}
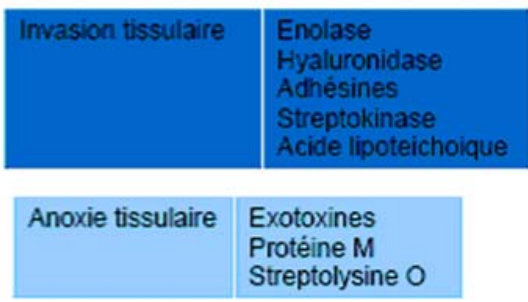

Fig. 1 Facteurs de virulence constitutionnels et sécrétés du streptocoque A $\beta$-hémolytique

d'adhésion tissulaire en se liant au fibrinogène et aux cellules épithéliales. Enfin, la formation d'un complexe protéine M1-immunoglobuline G (par l'intermédiaire du fragment Fc) induirait une activation plaquettaire et des thromboses [16]. De nombreuses études ont souhaité isoler le sérotype responsable des CTS, les sérotypes M1 suivi de M3 sont plus majoritairement retrouvés dans les CTS [14]. Malgré tout, d'autres sous-types de protéines M peuvent être responsables de la survenue d'un CTS [12].

L'acide lipoteichoïque possède une affinité pour la fibronectine qui favoriserait l'adhésion du germe au niveau des cellules épithéliales de la muqueuse buccale [17].

Enfin, le SAH possède de nombreuses autres adhésines (protéine $\mathrm{F}$ ou fibronectin binding protein, galactose binding protein, vitronectin binding protein, collagen binding protein...), qui possèdent des récepteurs spécifiques au niveau des cellules de l'hôte (fibronectine, vitronectine, collagène, plasmine). Ces adhésines permettent au SAH de se fixer sur les cellules épithéliales, les kératinocytes et d'autres cellules constituantes de l'hôte [18]. Cela explique la forte affinité du streptocoque pour les muqueuses (pharyngée, vaginale) et la peau.

\section{Facteurs de virulence sécrétés}

Le SAH sécrète des facteurs qui ont été reconnus comme des éléments de virulence du germe. Les exotoxines sécrétées par le SAH sont de deux grands types : les exotoxines 
pyrogéniques (A, B, C, D, I, J, L) et mitogéniques (SMEZ, SSA, SPE F, G, H, M) $[12,19]$. Ces toxines se comportent comme des superantigènes [20]. Les sous-types d'exotoxine favorisant la survenue d'un CTS sont variables $[12,14]$.

La streptolysine $S$ inhiberait l'action des polynucléaires neutrophiles.

La streptolysine $O$ inhiberait la phagocytose de la bactérie par les macrophages en accélérant leur apoptose [21]. Bryant et al. ont montré que la streptolysine $\mathrm{O}$ induirait un complexe entre des plaquettes et des polynucléaires neutrophiles et générerait des occlusions vasculaires qui aggraveraient l'anoxie tissulaire [22].

La streptokinase favorise la transformation de plasminogène en plasmine et la dissolution de la fibrine. Ainsi, elle favoriserait la dissolution tissulaire et donc l'invasion tissulaire de la bactérie. L'énolase aurait les mêmes propriétés [20].

La hyaluronidase, en détruisant le tissu conjonctif, serait un facteur de diffusion tissulaire du germe et de ses enzymes [15].

La C5a peptidase inactiverait une fraction C 5 du complément sérique [23].

Le streptococcus inhibitor of complement (SIC) permet au SAH d'inhiber la voie du complément et de résister au système de défense de l'hôte [24]. D'autres facteurs restent encore à découvrir.

\section{Mécanismes immunologiques}

\section{Superantigène et activation lymphocytaire}

L'exotoxine sécrétée par le SAH se comporte comme un superantigène $[1,9,20]$. Elle peut se fixer, d'une part, sur le récepteur des lymphocytes $\mathrm{T}$ (TCR) au niveau de la région variable, non spécifique $\beta$ et, d'autre part, sur le récepteur CMH (du système HLA II) de la cellule présentatrice d'antigène (CPA) (Fig. 2). La fixation d'un antigène spécifique n'est pas nécessaire à l'activation du lymphocyte T. La présentation de l'exotoxine au lymphocyte T (CD4) via la CPA engendre l'activation massive, non spécifique du lymphocyte $\mathrm{T}$ et la libération massive de cytokines et de facteurs de l'inflammation [20]. Les cytokines relarguées par les lymphocytes et par la CPA sont le TNF $\alpha$, le TNF $\beta$, l'IL-1, l'IL-2... Le TNF $\alpha$ est reconnu comme le médiateur du choc pour son action pro-inflammatoire, pyrogénique directe et indirecte, procoagulante et activateur de la sécrétion de catécholamines [19].

\section{Résistance aux défenses de l'hôte}

La sévérité du CTS est aggravée par les mécanismes de protection élaborés par le $\mathrm{SAH}$. Il résiste à la phagocytose grâce à sa capsule, aux protéines $\mathrm{M}$, et à la streptolysine $\mathrm{O}$.
(1) activation lymphocytaire spécifique par un antigène spécifique (2) activation lymphocytaire polyclonale non spécifique par l'exotoxine (superantigène) du Streptocoque $A \beta$ hémolytique

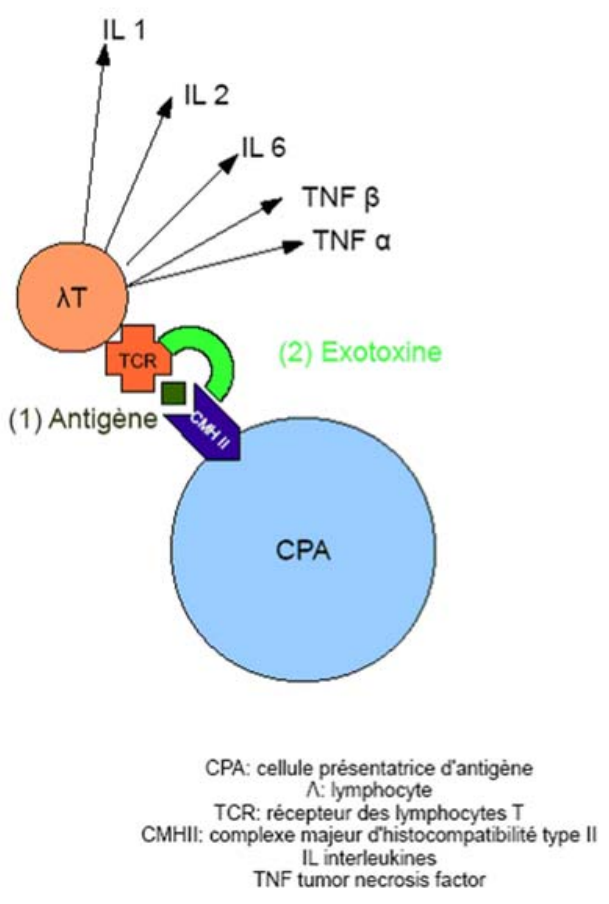

Fig. 2 Superantigène et activation lymphocytaire

Il possède une résistance au complément grâce la C5a peptidase, à une protéine SIC, à l'acide lipoteichoïque. De plus, lors de l'administration d'antibiotiques antiprotidiques, la destruction de la protéine $M$ s'accompagne d'un défaut d'immunisation du patient [1]. Enfin, il semblerait que le SAH peut générer la synthèse d'autoanticorps.

\section{Caractéristiques cliniques}

\section{Facteurs de risque}

Des facteurs de risque d'ISG et de CTS ont été identifiés dans plusieurs études [2,5,6,25] (Tableau 2). Dans $71 \%$ des ISG sont retrouvés un ou plusieurs facteurs de risque. Au cours de la varicelle ou d'une lésion cutanée, l'administration d'anti-inflammatoire non stéroïdien (AINS) augmenterait le risque de fasciite nécrosante [26]. Il semblerait que les AINS masqueraient les symptômes initiaux et engendreraient un retard diagnostique. Leur rôle sur la fonction granulocytaire et dans la production de cytokines prédisposerait au développement de formes graves. Au cours d'une varicelle, le risque de survenue d'un CTS doit être suspecté devant une fièvre supérieure à $39^{\circ} \mathrm{C}$ (au troisième jour du début de la maladie), une éruption devenue douloureuse, un érythème localisé chaud induré. 
Tableau 2 Critères diagnostiques du choc toxinique streptococcique d'après Breiman et al. [26]

Isolement d'une infection à streptocoque $\mathrm{A}$

Signes cliniques de sévérité

Hypotension

Adulte PAS $<90 \mathrm{mmHg}$

Enfant $<5^{\mathrm{e}}$ percentile pour l'âge

Plus de 2 signes suivants :

Insuffisance rénale

Et coagulopathie :

Plaquettes $<10000 \mathrm{~mm}^{3}$

Coagulation intravasculaire disséminée

Dysfonction hépatique avec élévation ASAT-ALAT

bilirubine

Syndrome de détresse respiratoire aigu

Érythème généralisé, rash cutané, nécrose des tissus mous

Les nombreux cas de CTS au cours du troisième trimestre de grossesse rapportés dans la littérature permettent d'isoler une nouvelle population à risque. Les cas décrits concernent des femmes jeunes en bonne santé et dont la grossesse ne présentait aucune complication. Dans la grande majorité des cas, le CTS a été fatal pour la mère et le fœtus [27].

Le contage familial est un facteur de risque de survenue d'un CTS. Le portage asymptomatique du SAH, notamment au niveau pharyngé, prédispose à une ISG [28].

\section{Présentation clinique}

Les symptômes initiaux sont peu spécifiques et simulent un syndrome grippal avec de la fièvre, des myalgies, des nausées ou des vomissements et des diarrhées aqueuses profuses. Des douleurs importantes ont été décrites au niveau du site infectieux. Une infection cutanée, gynécologique, pharyngée est le plus fréquemment à l'origine du CTS. Quatrevingts pour cent des CTS ont une infection des tissus mous, $70 \%$ ont une fasciite nécrosante et $10 \%$ ont un érythème scarlatiniforme associé $[1,11]$. Toutefois, il existe une grande variabilité des sites infectieux retrouvés au cours du CTS (méningite, empyème cérébral, pneumonie, arthrite septique, ostéomyélite, péritonite, endocardite, otite, endophtalmie, infection urinaire, infection puerpérale, nosocomiale...). En post-partum, la réalisation de geste gynéco-obstétrical aurait été incriminée dans la survenue d'un CTS. Mais il persiste de nombreux cas où le site infectieux initial n'a pas pu être identifié. Le contraste entre la bénignité apparente des symptômes initiaux et l'évolution rapidement sévère de l'infection est frappante. L'état de choc se révèle rapidement avec confusion, hypotension artérielle et dysfonction d'organes. L'évolution est fulgurante et le décès survient le plus souvent dans les 24 heures [11]. Le diagnostic de CTS implique une
Tableau 3 Facteurs de risque de choc toxinique streptococcique

Âge inférieur à 6 ans ou supérieur à 60 ans

Ethnie américaine ou africaine

Diabète

Séropositivité pour virus d'immunodéficience humaine (VIH)

Cancer, leucémie

Cardiopathie

Insuffisance rénale chronique

Alcoolisme chronique

Toxicomanie intraveineuse

Varicelle surtout en association aux anti-inflammatoire non stéroïdiens (AINS)

Prise d'AINS au début des symptômes

Grossesse $3^{\mathrm{e}}$ trimestre

instabilité hémodynamique associée au moins à deux défaillances d'organes (Tableau 3) [25].

À ce tableau classiquement décrit s'ajoutent quelques particularités sémiologiques : des cas d'hémorragies intraalvéolaires responsables d'une détresse respiratoire majeure sans coagulopathie préalable ont été décrits [27]. Le mécanisme reste non élucidé, le TNF $\alpha$ jouerait un rôle important dans la genèse de l'hémorragie intra-alvéolaire. La description de cas d'hémorragie intra-alvéolaire lors d'ISG, l'isolement de streptocoques dans les capillaires alvéolaires et l'absence d'autres causes retrouvées chez ces patients plaident en faveur de la responsabilité du germe. L'infection à SAH est une étiologie rare d'hémorragie intra-alvéolaire. Des embolies ou des abcès à distance ont été retrouvés, ce qui plaide pour le caractère emboligène du SAH. On a décrit des infarctus spléniques et pulmonaires [29], un CTS avec une phlébite iliofémorale [30] et une occlusion complète emboligène de l'artère brachiale droite ayant nécessité une amputation [31].

\section{Cas particulier du péripartum}

De relativement nombreux cas de CTS ont été rapportés chez la femme enceinte sans antécédent et permettent d'isoler une nouvelle population à risque [27]. Les cas décrits concernent exclusivement le troisième trimestre de la grossesse, en dehors de laquelle aucun facteur de risque n'était identifié. Les taux de mortalité maternelle et fœtale sont respectivement de 63 et $100 \%$ (Tableau 4). Le pronostic d'un CTS au troisième trimestre d'une grossesse reste effroyable malgré les traitements actuels. Le SAH a été isolé dans tous les cas, mais le site infectieux initial n'a pas toujours été identifié. Aucun geste gynéco-obstétrical n'avait été réalisé excluant ainsi une infection nosocomiale. 


\begin{tabular}{|c|c|c|c|c|}
\hline Études & Années de publication & Site infectieux & Mortalité maternelle & Mortalité fœtale \\
\hline Ooe et al. * & 1997 & Sang & Décès & Décès \\
\hline Ooe et al. * & 1997 & $\begin{array}{l}\text { Myomètre } \\
\text { Pharynx } \\
\text { Sang }\end{array}$ & Décès & Décès \\
\hline Ichiyama et al. * & 1997 & Sang & Décès & Décès \\
\hline Hirose et al. $*$ & 2001 & Sang & Décès & Inconnu \\
\hline Crum et al. * & 2002 & $\begin{array}{l}\text { Sang } \\
\text { Placenta }\end{array}$ & Survie & Décès \\
\hline Daif et al. ** & 2009 & $\begin{array}{l}\text { Sang } \\
\text { Muscle }\end{array}$ & Survie & inconnu \\
\hline
\end{tabular}

En post-partum immédiat, les nombreuses descriptions cliniques de la littérature montrent que le CTS existe [32-37]. L'origine du CTS est une infection génitale soit primitive (endométrite, pyométrite, mort fœtale in utero), soit secondaire à un geste obstétrical réalisé au moment de l'accouchement (épisiotomie, césarienne, forceps...). Trente pour cent de ces patientes sont décédées. Les quatre patientes ayant reçu des AINS avaient un taux de mortalité de $50 \%$.

L'évolution fulminante du CTS rend indispensable la reconnaissance et le diagnostic du CTS rapidement afin d'initier au plus tôt les traitements.

\section{Thérapeutiques}

\section{Support hémodynamique}

Le soutien de l'hémodynamique du patient est le premier traitement symptomatique instauré avant d'identifier l'origine de l'état de choc. Il nécessite une oxygénation, un remplissage vasculaire souvent important et le recours aux amines vasopressives.

\section{Antibiothérapie}

Le SAH est classiquement sensible aux $\beta$-lactamines, aux pénicillines et aux céphalosporines, mais quelques cas de résistances ont été décrits. Ces derniers semblent dépendre de l'environnement bactériologique et du sérotype M prépondérant [38]. In vitro, la pénicilline est l'antibiotique le plus efficace pour inhiber la croissance bactérienne. Toutefois, in vivo, cette efficacité est réduite du fait d'un « effet inoculum », et les fortes concentrations de germe dans les stades avancés du CTS mettent en défaut la pénicilline [39]. De plus, la précocité du traitement (avant la deuxième heure) influence l'efficacité de l'antibiotique. Le recours à des doses élevées d'antibiotiques le plus précocement possible est donc recommandé dans les ISG et le CTS [40]. La clindamycine a été utilisée en monothérapie ou bithérapie et a montré une supériorité par rapport à la pénicilline seule [41,42]. L'administration de clindamycine à forte dose a montré une réduction de la mortalité $(25$ à $40 \mathrm{mg} / \mathrm{kg}$ par jour) $[41,42]$. L'effet dose semble prépondérant dans l'efficacité du traitement. In vitro, la clindamycine inhiberait la production d'exotoxines, la synthèse de la protéine $M$ et la sécrétion de TNF $\alpha$. Quelques cas de résistances à la clindamycine ont été décrits. Le mécanisme reste incertain, mais l'étude de Minami et al. a montré une augmentation de la production de trois exotoxines (streptolysine O, NAD glycohydrolase, streptokinase) secondaire à l'expression d'un ARN messager, CovS, chez les souches résistantes à la clindamycine [41]. L'association de pénicilline et de clindamycine n'a pas montré de supériorité par rapport à une monothérapie. Le recours à une bithérapie n'est donc pas recommandé à l'heure actuelle. Le linezoline possède la même efficacité que la clindamycine. Il inhiberait la synthèse protidique, et son efficacité serait durable [42].

\section{Immunoglobulines}

Une réduction de mortalité a été observée après administration de gamma-immunoglobulines (IgG) polyspécifiques [43]. Le mécanisme d'action n'est pas élucidé, mais les IgG pourraient bloquer l'activation lymphocytaire induite par le superantigène et la sécrétion de cytokines. Les IgG polyspécifiques contiendraient des anticorps spécifiques des superantigènes (anti-SpeC) [43]. Les différentes préparations d' $\operatorname{IgG}$ existantes ont un effet neutralisant spécifique et variable en fonction des anticorps antisuperantigènes contenus. Leur efficacité est donc variable en fonction de la préparation disponible [43]. 


\section{Chirurgie}

La fasciite nécrosante ou la myosite sont des urgences chirurgicales. La précocité de la détersion et du débridement limite la progression en profondeur de l'infection et permet d'éviter les délabrements importants et/ou les amputations [9,14,39]. Dans les cas de myométrite, l'hystérectomie a été salvatrice [28]. Même en cas de généralisation de l'infection et de CTS, la suppression chirurgicale du foyer infectieux est indispensable. Dans les cas de CTS sans site infectieux retrouvé et/ou avec bactériémie isolée, la chirurgie n'est d'aucun recours.

\section{Prévention et vaccination}

Les données épidémiologiques mettent en évidence une contamination de proche en proche, notamment au sein de la famille. De nombreux individus sont porteurs sains au niveau pharyngé. Certains auteurs ont proposé l'éradication préventive de ce portage par l'administration de pénicilline associée à la rifampicine ou de clindamycine en monothérapie $[44,45]$.

L'émergence des ISG, leur sévérité, l'incapacité des traitements à réduire la mortalité et la richesse antigénique du streptocoque pyogène favorisent le développement d'un vaccin. Le vaccin anti-SAH est actuellement en phase I d'essai clinique. Mais la variabilité des structures antigéniques retrouvées au cours du CTS, non seulement d'un patient à l'autre, mais aussi au cours du temps et en fonction du pays considéré, rendent difficile sa conception $[46,47]$. La protéine $M$ reste l'élément central du vaccin, mais l'absence de spécificité de sous-types M au cours du CTS multiplie le nombre de valences nécessaires à une bonne couverture vaccinale. Les 26 valences contenues dans un vaccin américain ne couvrent que $60 \%$ des streptocoques responsables de CTS [48].

\section{Conclusion}

Le CTS est une pathologie soudaine, rapidement sévère associée à un taux de mortalité important. Les mécanismes physiopathologiques sont complexes. Après l'invasion, le SAH provoque le relargage massif de cytokines et résiste aux défenses immunitaires de l'hôte, générant ainsi un état de choc toxinique non contrôlé. Le CTS ne supporte aucun retard diagnostique. Il est primordial de connaître les particularités sémiologiques du CTS afin de le reconnaitre précocement. Le péripartum est une période à risque, où l'infection à SAH doit être suspectée devant tout sepsis. Le traitement doit être précoce et agressif afin d'améliorer le pronostic vital du patient. Un vaccin, actuellement en cours de développement, devrait permettre de réduire l'incidence et la mortalité du CTS.

Conflits d'intérêt : les auteurs ne déclarent pas de conflit d'intérêt.

\section{Références}

1. Stevens DL (2002) Streptococcal toxic-shock syndrome. Clin Microbiol Infect 8:133-6

2. Davies H, McGeer A, Schwartz B, et al (1996) Invasive group A streptococcal infections in Ontario, Canada. N Engl J Med 335:547-54

3. Darenberg J, Luca-Harari B, Jasir A, et al (2007) Molecular and clinical characteristics of invasive group A streptococcal infection in Sweden. Clin Infect Dis 45:450-8

4. Ekelund K, Skinhoj P, Madsen J, et al (2005) Invasive group A, B and G streptococcal infection in Denmark 1999-2002: epidemiological and clinical aspects. Clin Microbiol Infect 11:569-76

5. Lamagni T, Darenberg J, Luca-Harari B, et al (2008) Epidemiology of severe streptococcus pyogenes disease in Europe. J Clin Microbiol 46:2359-67

6. O'Brien K, Beall B, Barret P, et al (2002) Epidemiology of invasive group A streptococcus disease in the united states, 19951999. Clin Infect Dis 35:268-76

7. O'Grady K, Kelpie L, Andrews RM, et al (2007) The epidemiology of invasive group A streptococcal disease in Victoria, Australia. Med J Aust 186:565-9

8. Martin PR, Hoiby EA (1990) Streptococcal serogroup A epidemic in Norway 1987-1988. Scand J Infect Dis 22:421-9

9. Stevens DL (1992) Invasive group A streptococcus infections. Clin Infect Dis 14:2-11

10. Demers B, Simor AE, Vellend H, et al (1993) Severe invasive group A streptococcal infections in Ontario, Canada 1987-1991. Clin Infect Dis 16:792-800

11. Baxter F, McChesney J (2000) Severe group A streptococcal infection and streptococcal toxic shock syndrome. Can J Anaesth 47:1129-40

12. Stanley J, Linton D, Desai M, et al (1995) Molecular subtyping of prevalent $\mathrm{M}$ serotypes of streptococcus pyogenes causing invasive disease. J Clin Microbiol 33:2850-5

13. Mehta S, McGeer A, Low DE, et al (2006) Morbidity and mortality of patients with invasive group A streptococcal infections admitted to the ICU. Chest 130:1679-86

14. Brown EJ (2004) The molecular basis of streptococcal toxic shock syndrome. N Engl J Med 350:2093-4

15. Starr CR, Engleberg NC (2006) Role of hyaluronidase in subcutaneous spread and growth of group A streptococcus. Infect Immun $74: 40-8$

16. Shannon O, Hertzen E, Noorby-Teglund A, et al (2007) Severe streptococcal infection is associated with $M$ protein-induced platelet activation and thrombus formation. Mol Microbiol 65:1147-57

17. Courtney HS, Ofek I, Penfound T, et al (2009) Relationship between expression of the family of $\mathrm{M}$ proteins and lipoteichoic acid to hydrophobicity and biofilm formation in streptococcus pyogenes. PLoS One 4(1):e4166

18. Gorton D, Norton R, Layton R, et al (2005) Presence of fibronectin-binding protein gene $p r t F 2$ in invasive group A streptococci in tropical Australia is associated with increase internalisation efficiency. Microbes Infect 7:421-6 
19. Cunningham MW (2000) Pathogenesis of group A streptococcal infections. Clin Microbiol Rev 13:470-511

20. Thomas D, Perpoint T, Dauwalder O, et al (2009) In vivo and in vitro detection of a superantigenic toxin Vbeta signature in two forms of streptococcal toxic shock syndrome. Eur J Clin Microbiol Infect Dis 28:671-6

21. Timmer AM, Timmer JC, Pence MA, et al (2009) Streptolysin O promotes group A streptococcus immune evasion by acceleration macrophage apoptosis. J Biol Chem 284:862-71

22. Bryant AE, Bayer CR, Chen RY, et al (2005) Vascular dysfunction and ischemic destruction of tissue in streptococcus pyogenes infection: the role of streptolysinO-induced platelet/neutrophil complexes. J Infect Dis 192:1014-22

23. O'Connor SP, Cleary P (1986) Localization of the streptococcal C5a peptidase to the surface of group A streptococci. Infect Immun 53:432-4

24. Frick IM, Akesson P, Rasmussen M, et al (2003) SIC, a secreted protein of streptococcus pyogenes that inactivates antibacterial peptides. J Biol Chem 278:16561-6

25. Montgomery VL, Bratcher D (1996) Complications associated with severe invasive streptococcal syndrome. J Pediatr 129:602-4

26. 1993) Defining the group A streptococcal toxic shock syndrome. Rationale and consensus definition. The Working Group on Severe Streptococcal Infections JAMA 269:390-1

27. Hamilton SM, Bayer CR, Stevens DL, et al (2008) Muscle injury, vimentin expression and nonsteroidal anti-inflammatory drugs predispose to cryptic group A streptococcal necrotizing infection. J Infect Dis 198:1692-8

28. Crum NF, Chun HM, Gaylord TG, et al (2002) Group A streptococcal toxic shock syndrome developing in the third trimester of pregnancy. Infect Dis Obstet Gynecol 10:209-16

29. Barnham MR, Weightman NC (2001) Bacteraemic streptococcus pyogenes infection in the peripartum period: now a rare disease and prior carriage by the patient may be important. J Infect 43:173-6

30. Torda A (2005) Post-partum toxic shock syndrome associated with multiple splenic infarcts. Med J 182:93

31. Verwoerd GR, Gebhardt GS (2004) Group A beta-hemolytic streptococcal toxic shock syndrome presenting as suspected ileo-femoral thrombosis in the puerperium. J Obstet Gynecol 24:934

32. Susset MA, Sczepanski B, Herrmann M, et al (1998) Puerperal sepsis caused by streptococcus group A with a severe form of progression like "toxic shock like syndrome". Dtsch Med Wochenschr 123:588-93

33. Jorup-Rönström C, Hofling M, Lundberg C, et al (1996) Streptococcal toxic shock syndrome in a post-partum woman. Case report and review of the literature. Infection 24:164-7

34. Golden S (2003) Group A streptococcus and streptococcal toxic shock syndrome: a post-partum case report. J Midwifery Womens Health 48:357-9
35. Daif JL, Levie M, Chudnoff S, et al (2009) Group A streptococcus causing necrotizing fasciitis and toxic shock syndrome after medical termination of pregnancy. Obstet Gynecol 113:504-6

36. Schummer W, Schummer C (2002) Two cases of delayed diagnosis of postpartal streptococcal toxic shock syndrome. Infect Dis Obstet Gynecol 10:217-22

37. Feigenberg T, Sela HY, Applebaum YH, et al (2008) Puerperal widespread pyomyositis after group A streptococcal toxic shock syndrome. Isr Med Assoc J 10:483-4

38. Castagnola DE, Hoffman MK, Carlson J, et al (2008) Necrotizing cervical and uterine infection in the post-partum period caused by group A streptococcus. Obstet Gynecol 111:533-5

39. Capoor MR, Nair D, Deb M, et al (2006) Resistance to erythromycin and rising penicillin MIC in streptococcus pyogenes in Indian. Jpn J Infect Dis 59:334-6

40. Stevens DL, Gibbons AE, Bergstrom R, et al (1988) The Eagle effect revisited: efficacity of clindamycin, erythromycin and penicillin in the treatment of streptococcal myositis. J Infect Dis 158:23-8

41. Stevens DL (2003) Dilemmas in the treatment of invasive streptococcus pyogenes infections. Clin Infect Dis 37:341-3

42. Minami M, Kamimura T, Isaka M, et al (2010) Clindamycininduced CovS-mediated regulation of the production of the virulent exoproteins streptolysin A, NAD glycohydrolase and streptokinase in streptococcus pyogenes. Antimicrob Agents Chemother 54:98-102

43. Coyle EA, Cha R, Rybak MJ (2003) Influences of linezolid, penicillin and clindamycin, alone and in combination on streptococcal pyrogenic exotoxin a release. Antimicrob Agents Chemother 47:1752-5

44. Norrby-Teglund A, Haque KN, Hammarström L (2006) Intravenous polyclonal IgM-enriched immunoglobulin therapy in sepsis: a review of clinical efficacy in relation to microbiological etiology and severity of sepsis. J Intern Med 260:509-16

45. Tanz RR, Schulma, ST, Barthel MJ, et al (1985) Penicillin plus rifampicin eradicates pharyngeal carriage of group A streptococci. J Pediatr 106:876-80

46. Tanz RR, Poncher JR, Coryndon KE, et al (1991) Clindamycin treatment of chronic pharyngeal carriage of group A streptococci. J Pediatr 119:123-8

47. Nir-Paz R, Korenman Z, Ron M, et al (2010) Streptococcus pyogenes emm and $T$ types within a decade, 1996-2005: implications for epidemiology and future vaccines. Epidemiol Infect 138:53-60

48. Sterr AC, Law I, Matatolu L, et al (2009) Global emm type distribution of group A streptococci: systematic review and implications for vaccine development. Lancet Infect Dis 9:611-6

49. Kaplan EL, Wotton JT (2001) Dynamic epidemiology of group A streptococcal serotypes associated with pharyngitis. Lancet 358:1334-7 